\title{
Driving Simulator for Performance Monitoring with Physiological Sensors
}

\author{
Diogo Raimundo \\ Instituto Politécnico de Lisboa (IPL) \\ Instituto Superior de Engenharia de Lisboa (ISEL) \\ Email: a38429@alunos.isel.pt
}

\author{
André Lourenço \\ CardioID Technologies \\ Instituto Politécnico de Lisboa - ISEL \\ Instituto de Telecomunicações (IT) \\ Email: arl@cardio-id.com
}

\author{
Arnaldo Abrantes \\ Instituto Politécnico de Lisboa \\ ISEL \\ Email: aja@deetc.isel.pt
}

\begin{abstract}
This paper describes a driving monitoring system based on a car driving simulator, specifically built for this project, using inexpensive off-the-shelf game technology (software and hardware). The project aims to study the effect of drowsiness (caused by fatigue, alcohol consumption, etc.) in driving performance and how it can be detected in advance in order to mitigate accidents. To achieve this, the system is continuously monitoring driver's physiological signals (e.g., heart rate) as well as her/his driving behavior. Electrocardiography (ECG) signal plays a central role in this project, since a feature derived from it - the HRV (Heart Rate Variability), more concretely its power spectral distribution - is used to continuously estimate the driver's degree of awareness and therefore for generation of alarms. A preliminary evaluation of the proposed system is discussed through the presentation of some experimental results.
\end{abstract}

\section{INTRODUCTION}

High levels of driver fatigue or sleepiness is surely one of the main causes of road accidents in the world [1]. In fact, it is estimated that in the United States of America, between 2005 and 2007, 94\% of the motor vehicle crashes occur due to human error, where $41 \%$ of these crashes are assigned to recognition error, which include lack of attention, internal and external distractions, and inadequate surveillance [2]. It should be noted that although only $7 \%$ of the crashes are directly assigned to falling asleep on the wheel, accidents due to recognition $(41 \%)$, decision $(33 \%)$ or performance $(11 \%)$ errors may also be caused by driver fatigue or sleepiness. Moreover, this problem may also be fostered by the consumption of alcohol and psychotropic substances, which constitute a big "threat" to the good performance of drivers.

Studying the effect of sleepiness (drowsiness) on driving is therefore an important research topic, being the usage of driving simulators an appealing strategy as they provide a controlled and repeatable environment to perform tests on a sample of subjects. However, these simulators tend to be complex and monetarily expensive [3], and moreover the results may also not represent true situations due to the lack of realism of the virtual environment. In addition, the driver is aware that he is being evaluated and may try to alter his behavior, even in a involuntary manner.

On the other hand, road driving tests are a generally less costly approach and may result in better and more objective measures. However, in this case, legal and ethical reasons strongly limit the range of tests that can be performed, when compared to the use of a driving simulator, as it is obviously unacceptable to put persons in danger. Besides, controllable and repeatable road driving tests are difficult, if not impossible, to put in practice.

Whether opting to use a driving simulator or not, there is a need to monitor the driver by acquiring and analyzing sensory data in many physical and emotional conditions, in order to investigate the correlations between those conditions and the corresponding performances. Among all the sensory data that can be acquired regarding the drowsiness state of a driver, some studies [1] highlight the use of electrocardiogram (ECG) and electroencephalogram (EEG) [4], [5] as the most accurate approach to detect fatigue.

Other measures that may be used are [1]:

- Behavioral measures such as head pose, yawning, eye closure or even eye blinking;

- Performance measures such as decrease of micromovements on the steering wheel or deviation from lane position;

- Subject self-measures using the Karolinska sleepiness scale (KSS).

This paper describes a platform, based on a driving simulator, that can be used to acquire and process sensory data, in particular heart-rate variability (HRV), steering wheel movement (SWM) and standard deviation of lateral position (SDLP) [6], [7].

The remaining sections of the paper are organized as follows. The next section puts our work in context by relating it with others, section III presents the physiological signals and sensors used in our system while section IV describes the architecture of the system. In section V, some experimental results will be presented and discussed, and finally, section VI concludes the paper.

\section{RELATED WORK}

Driving simulators are usually classified in low, mid or high level, according to their fidelity to the reality. A low-level setup would have a steering wheel and pedals, a realistic cockpit and one to three monitors. If it uses advanced imaging techniques (e.g. large projection screen), a realistic car and even a simple 
motion base, it is classified as a mid-level setup. Finally, to be considered a high-level driving simulator, it should provide a $360^{\circ}$ view and an advanced moving base [8].

A simulated experience must always be validated to assess if its results are transferable to the reality, i.e., if a real experience will obtain the same results under the same circumstances [8]. There are multiple ways to achieve this validation, being one the Face validity, which basically evaluates how realistic is the experimental environment.

A very similar study from Instituto de Biomecânica de Valência [9] used a low-level simulator in a controlled room to induce and measure drowsiness in a small sleep-deprived group of subjects. By using electrocardiography (ECG), electroencephalography (EEG), electrooculography (EOG), driving performance and the percentage of eyelid closure (PERCLOS) [10], the researchers were able to easily discriminate attentive drivers from drowsy ones, but not if they were only fatigued.

\section{SENSORS AND SIGNALS}

Since the beginning driver's performance measures, and vehicle-based measures, have been considered poor discriminating features for driver drowsiness as they are not specific enough: a driver under the influence of alcohol or others drugs would have a similar result as it was drowsy. Nevertheless, this approach to measure driver drowsiness have been broadly used [7], being one of the most common the steering wheel movement (SWM). When drowsy, the driver involuntarily reduces the number of micro-corrections (between 0.5 and 5 degrees) on the steering wheel. However, this measure can be difficult to use in some situations as it is very dependent on the road geometry. As the SWM reduces, the vehicle's lane position oscillates with increasing amplitudes and the measurement of these values can then be used to compute the standard deviation of lane position (SDLP), which is another measure that is widely used to evaluate the level of driver drowsiness. Like SWM, this measure also have significant dependency on the subject and on the road conditions, although its results has been correlated to KSS ratings.

A major problem of only using performance measures to detect fatigue is that it becomes detectable too late, when it is already influencing the driver's actions, and therefore may be unable to prevent a car crash. In contrast, by being directly acquired from the driver's body, physiological measures can show drowsiness evidence when it is still in early stages, making them more suitable to detect and notify in advance a drowsy driver in order to prevent a possible accident. However, one must be aware that this type of measurements increases significantly the complexity of the experimental setup as these (physiological) signals are extremely low energy signals and therefore very susceptible to be distorted by environment noise, which implies the use of sophisticated signal conditioning and filtering operations. These processed signals are then used to compute a set of features which in turn will be ultimately used as the input of a classifier to evaluate the driver's state [1], [11].

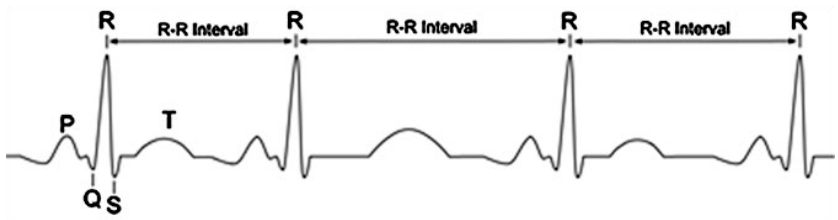

Fig. 1. Simulation results for the network

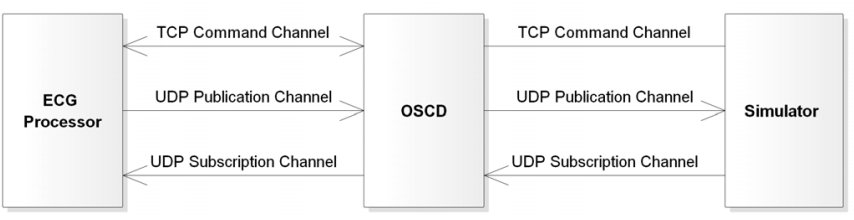

Fig. 2. OSC channels over transport protocol

The present work has a special focus on the ECG signal, and more specifically on a feature extracted from this signal, the heart-rate variability (HRV), which describes time variations in the RR intervals (see figure 1). Usually a frequencydomain analysis of this feature is performed by computing its power spectral density (PSD), which can be used to obtain a reliable measure of the sympathetic and parasympathetic driver's systems activity, and therefore detect the transition from being awake to be sleeping.

In fact, the sympathetic nervous system's activity, known to respond to stress situations such as fight or flight, is related to an individual's alert state, and can be measured on the lowfrequency band $(\mathrm{LF}=[0.04,0.15[\mathrm{~Hz})$ of the HRV signal. On the other hand, the parasympathetic nervous system, known to be responsible for decreasing the heart rate and blood pressure, increases its activity while the individual is in a relaxed state of mind [12], and can be measured on the high-frequency band $(\mathrm{HF}=[0.15,0.4] \mathrm{Hz})$ of the HRV signal [6], [11]. The ratio between the low-frequency and high-frequency PSD of the HRV signal is used as a detection measure of the transition from being "awake" to be "sleeping".

\section{SySTEM OVERVIEW}

This article focus on the building of a extensible platform that can acquire physiological and performance measures and it is planned to be used to acquire data in two scenarios: with drivers in sleep deprivation and under the influence of alcohol (DUI). It is important to acquire data of the second scenario because it has been shown that the effects of drowsy driving are very similar to driving under the influence of alcohol [13], [14].

The system was developed in three modules: Simulator, ECG processing, and Data distribution, as depicted in figure 2.

One core novelty of this simulator is the inclusion of an ECG sensor embedded into the steering wheel, integrating a custom version of CardioWheel being developed by CardioID Technologies [15], that was adapted to the driver simulator steering wheel. 


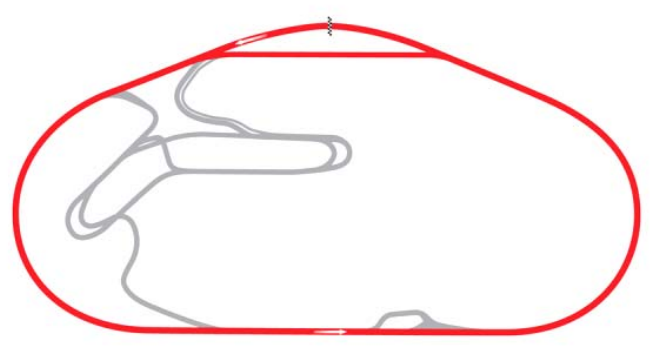

Fig. 3. Daytona International Speedway Tri-Oval Layout

\section{A. Simulator}

The simulator was developed using the Unity engine platform. This choice opens us the possibility to use a large number of free assets from its Standard Assets package, including the vehicle controller which plays a central role in this project. The simulation environment was designed as a simple race track that, without opponents, could be monotonous enough to cause fatigue.

Once the wheel embodies the most important physiological signal used in the project, the track was designed in order to reduce the necessity of taking the hands out of the wheel or rubbing them on it. These precautions are necessary because the rubbing movement adds noise to the acquired ECG signal, which then makes more difficult to extract the required features. Taking the hands out of the wheel, even if just one of them, will obviously cause a disconnection between the system and the driver that interrupts acquisition of physiological signals. Using an undemanding track is therefore mandatory for the success of the experiments. The track chosen is based on the Tri-Oval layout of the Daytona International Speedway, fine-tuned using Blender, which has a simple layout with open curves but not as monotonous as it would be a trivial oval shape track (see figures 3 and 4).

To improve the driver immersion in the activity, the track is surrounded by trees, the ground was raised in the center to better emulate a natural valley scenario, and a lake skybox is used for modeling the distant landscape. In order to prevent the driver from leaving the asphalt, the track was surrounded by cement barriers that were also modeled and textured specifically for the project in Blender.

As an approach to introduce "traffic" in the simulation, it was developed a package named Boids that implements a swarm intelligence [16] model with the same name [17], developed by Craig Reynolds in 1986, which enables a population of autonomous agents to simulate a flocking behavior, using a blending of 3 separate rules: separation, alignment and cohesion. Although this behavior does not seem to be suitable for the task of steering accurately a group of virtual cars, by giving one of them, the leader, a target to reach or a

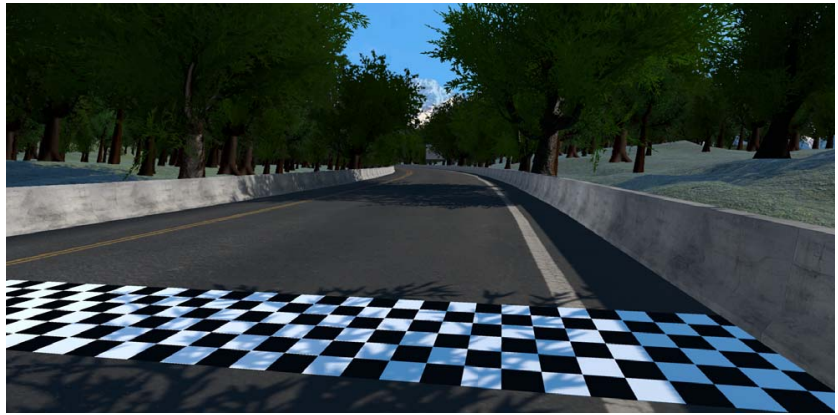

Fig. 4. Modeled environment (perspective view)

path to follow, it may be used to introduce some novelty and unpredictability to the simulation, forcing the driver to make decisions, which can be evaluated continuously by the system (e.g., braking time, number of collisions) and subsequently correlating the obtained score with the corresponding physiological measured states. Other realistic autonomous agent behaviors, like wander, pursuit, etc., can be added to increase the complexity and randomness of the scenario [18].

As mentioned before, one of the contributors for selecting Unity was the large amount of free assets existent. In particular, its vehicles controllers package was a big time saver for already implementing a basic car controller with all the basic physics needed, although a very erratic. One of the challenges of using the Boids package developed was that each update produces a arbitrary force vector. On the other hand the included car controller receives an input as four values between 1 and -1: steering, accelerator, footbrake and handbrake.

To translate the vector information to decimal values, it was defined sensitivity multipliers for the acceleration and steering inputs. The first value is obtain by calculating the magnitude of the force vector, weighted by its sensitivity multiplier, and then clamp it between the 1 and -1 . This value is used as accelerator and footbrake inputs since a code analysis revealed that this was the expected behaviour of the controller. To calculate the steering input, on the other hand, it was necessary to compute the angle between the generated force vector and the current forward vector of the car, using the arctangent. After multiplying it by the steering sensitivity value and clamping the result between 1 and -1 , the value is used as steering input on the controller.

\section{B. ECG Processing}

The monitoring of the ECG signal is performed using CardioID's technology, enabling the acquisition of this signal using only the hands of the driver, and is illustrated in Figure 5. The electrodes are based on conductive materials, and are connected to a embedded system with a custom analog front end that prepares the signal for analog to digital conversion, and also provides a lead-on detection signal. For convenience the signal is transmitted to the OSC server using a Bluetooth model. 


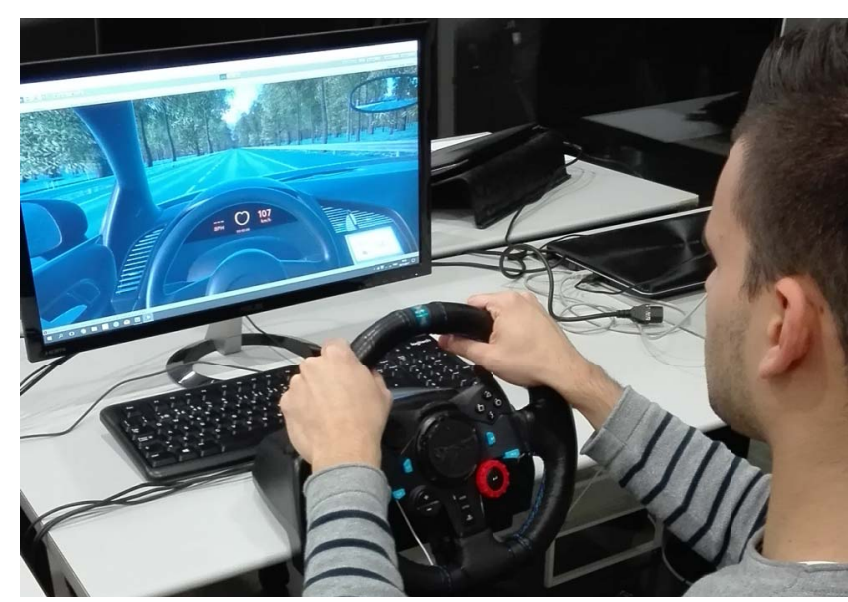

Fig. 5. Prototype of the Steering Wheel.

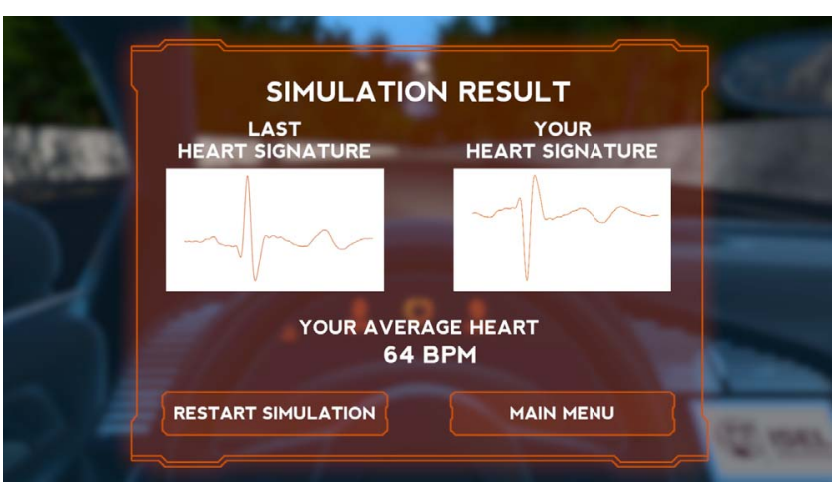

Fig. 6. Simulation report

The ECG is processed using the standard patternrecognition workflow: i) band-pass filtering; ii) R-peak detection; iii) HRV feature extration.

There are two primary approaches for the analysis of HRV and both are implemented on this work: time domain and frequency domain methods. On the time domain, the normalto-normal $(\mathrm{NN})$ interval (the interval between adjacent normal QRS complexes) or the instantaneous heart rate (heart rate calculated on a beat by beat basis) is determined and simple descriptive time domain variables such as the mean NN interval, mean heart rate, and the range (longest $\mathrm{NN}$ minus the shortest $\mathrm{NN}$ ) for a given time interval were calculated. Additionally the square root of the variance of the NN interval (SDNN) was also calculated. For the frequency perspective we used Power Spectral Density (PSD) estimation, enabling the calculation of VLF, LF and HF bands, and the ratio LF/HF.

Only for informative purposes, the driver's heart rate is continuously displayed on the front panel of the car during the simulation, as well as an indication whether the hands are being detected, or not, on the steering wheel (based on lead-on detection). Two alerts are also displayed on the front panel, triggered by fatigue detection and driver change, respectively. When the simulation ends, it is also displayed a ECG template of the current and previous driver, as illustrated in figure 6 .

\section{Data Distribution}

The last module concerns to data distribution. The collected data consists of momentary events (e.g., get off the road) and the measurements of the driver's performance, computed using \pm 17 millisecond windows $(60 \mathrm{~Hz})$. There are 6 performance measures being computed: throttle and brake movement, lane position and angle deviation, SWM and velocity. The first two signals are described by their derivatives and the other by their means and standard deviations. In addition, the system triggers events when the driver touches the lateral boundaries and when passes through the finish line. All this data is continuously sent to the data distribution server to be stored.

The data distribution module, named OSC Distributor (OSCD), was necessary to coordinate all communications between the other modules and to allow the system to be easily scalable. As communication protocol, it uses an TCP and UDP implementation of the Open Sound Control (OSC) and the data distribution feature is implemented based on the PublishSubscribe integration pattern [19], [20]. The OSC protocol was chosen due to its simple yet robust architecture, which allows interoperability, precision and flexibility, being the last one due to its address pattern that allows hierarchical structure.

Using this address pattern as subject description, this distribution server uses a dynamic implementation of the PublishSubscribe integration pattern to manage users, publications, subscriptions and to distribute the data published after the client configuration. To send and receive all this management commands, the system uses a dedicated OSC over TCP message protocol that also uses OSC addresses as descriptors of what action a given command refers to (see figure 2).

Before being capable of publishing or subscribing any subject, a client must first use the RegisterClient command to become part of the system. After this first step, the client can then register subjects, that will later be used to publish information, or subscribe to other subjects already existent on the system. To publish information, the client must send it using OSC over a UDP channel (see figure 2) to the endpoint given by the distribution server at the moment of registering the subject.

If a client tries to subscribe a subject that does not yet exist on the system, it can enable a notification alert for when it becomes available to subscribe. Similarly, if the client it is already subscribed to a subject that becomes unavailable, the distribution server sends a cancellation alert to it.

\section{Preliminary Evaluation}

The several components of the system were evaluated isolated in order to guarantee the integrity of the results. Figure 10 presents an example of the RR series obtained during one of the simulations, while figures 7,8 and 9 present steering wheel movement based measures plus lane switching and collision events.

Based on this measures a set of acquisition under different conditions is under analsysis: i) normal condition; ii) Driver sleep Deprivation; iii) Influence of Alcohol. Preliminary analysis shows promising results. 

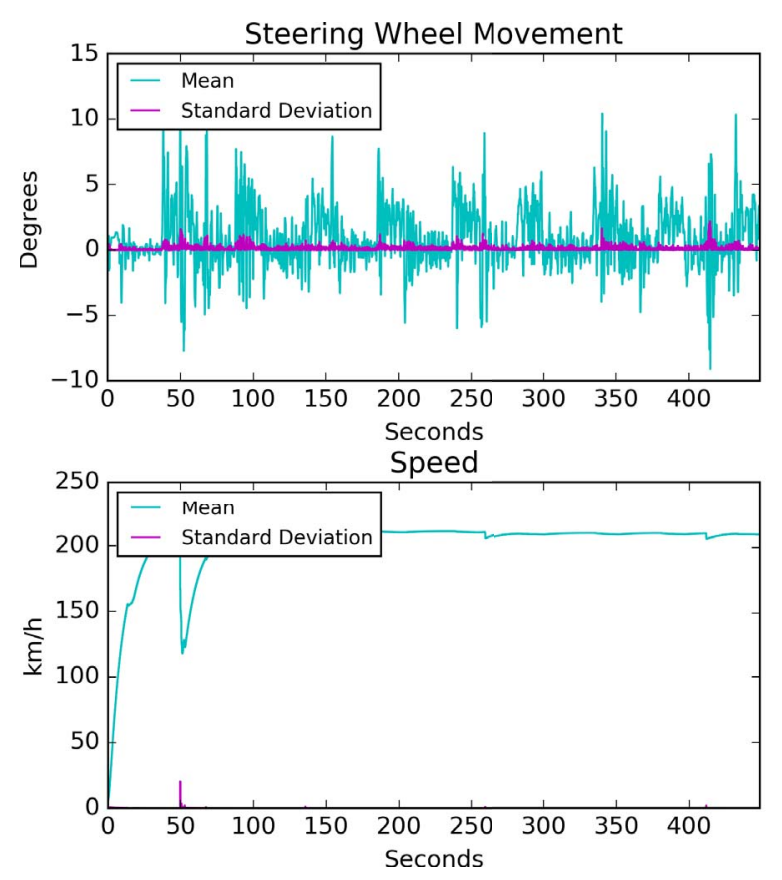

Fig. 7. Performance - SWM and Speed
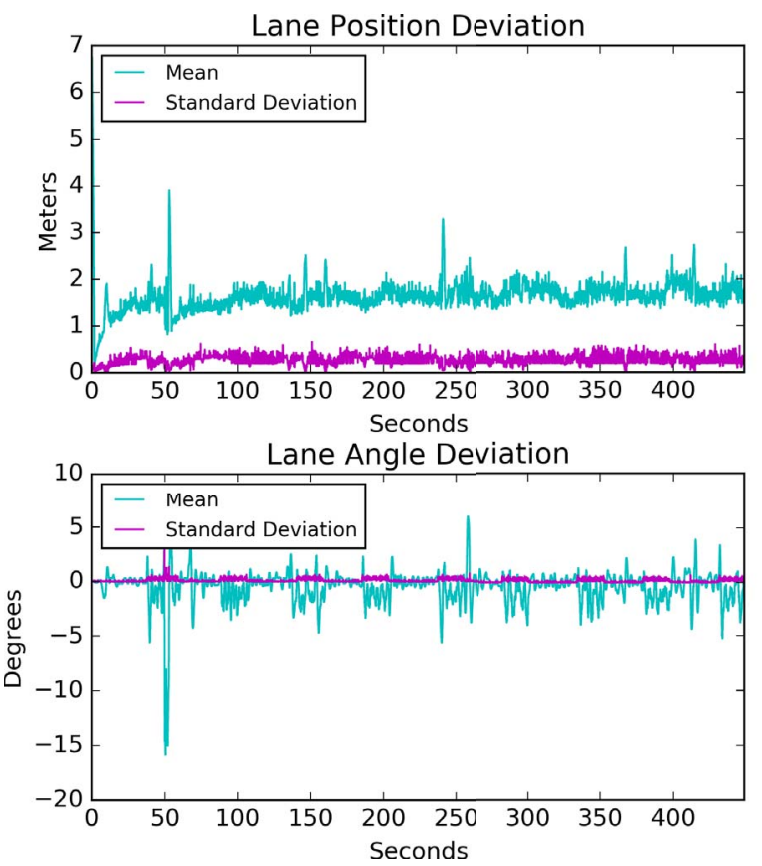

Fig. 8. Performance - LPD and LAD

\section{CONCLUSiOn AND Future Work}

Driver simulators constitute one of the tools used for the study and validation of driver monitoring solutions. Steering Wheel movements (SWM) and ECG signals were the two inputs for a platform based on Unity. The solution was developed thinking in its extensible to other inputs, using the OSC protocol, and in the near future will include other sensors a the Intel Real Sense Camera for face tracking and eye lid

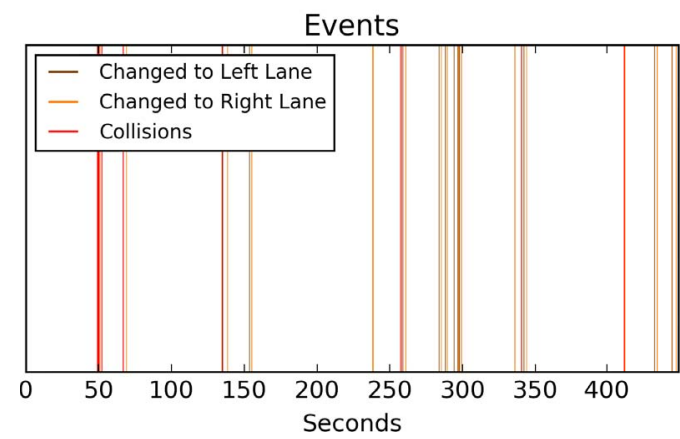

Fig. 9. Performance - Simulation Events

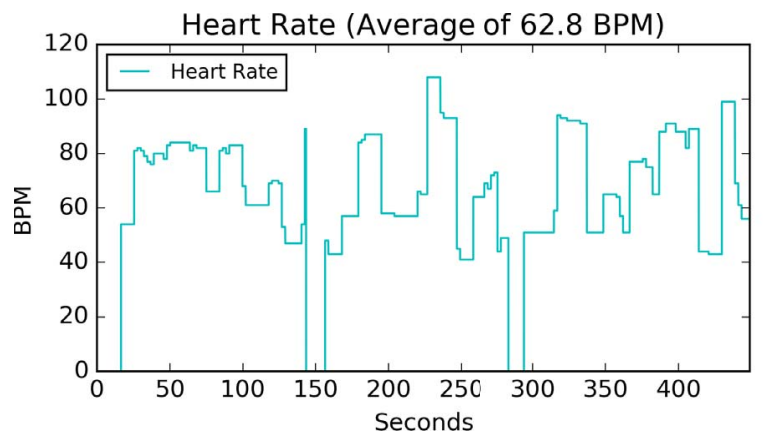

Fig. 10. Heart Rate

closure analysis.

These solutions have a tremendous importance for the automotive industry and are becoming part of the advanced driver assisting systems (ADAS) which are helping to reduce the number of accidents and helping improve safety on roads.

\section{REFERENCES}

[1] A. Sahayadhas, K. Sundaraj, and M. Murugappan, "Detecting driver drowsiness based on sensors: a review," Sensors, vol. 12, no. 12, pp. 16937-16953, 2012.

[2] S. Singh. (2015, February) Traffic safety facts - critical reasons for crashes investigated in the national motor vehicle crash causation survey. [Online]. Available: crashstats.nhtsa.dot.gov/Api/Public/ViewPublication/812115

[3] K. C. Baldwin, D. D. Duncan, and S. K. West, "The driver monitor system: A means of assessing driver performance," Johns Hopkins APL Technical Digest, vol. 25, no. 3, pp. 269-277, 2004.

[4] M. Akin, M. B. Kurt, N. Sezgin, and M. Bayram, "Estimating vigilance level by using eeg and emg signals," Neural Computing and Applications, vol. 17, no. 3, pp. 227-236, 2008.

[5] A. Kokonozi, E. Michail, I. Chouvarda, and N. Maglaveras, "A study of heart rate and brain system complexity and their interaction in sleepdeprived subjects," in Computers in Cardiology, 2008. IEEE, 2008, pp. 969-971.

[6] W. C. Liang, J. Yuan, D. C. Sun, and M. H. Lin, "Changes in physiological parameters induced by indoor simulated driving: Effect of lower body exercise at mid-term break," Sensors, vol. 9, no. 9, pp. 6913-6933, 2009. [Online]. Available: http://www.mdpi.com/1424$8220 / 9 / 9 / 6913$

[7] C. C. Liu, S. G. Hosking, and M. G. Lenné, "Predicting driver drowsiness using vehicle measures: Recent insights and future challenges," Journal of safety research, vol. 40, no. 4, pp. 239-245, 2009.

[8] N. Kaptein, J. Theeuwes, and R. Van Der Horst, "Driving simulator validity: Some considerations," Transportation Research Record: Journal of the Transportation Research Board, no. 1550, pp. 30-36, 1996. 
[9] H. D. Rosario, J. S. Solaz, N. Rodríguez, and L. M. Bergasa, "Controlled inducement and measurement of drowsiness in a driving simulator," 2010. [Online]. Available: http://harken.ibv.org/index.php/documents/doc_download/80-controlledinducement-and-measurement-of-drowsiness-in-a-driving-simulator

[10] D. Sommer and M. Golz, "Evaluation of perclos based current fatigue monitoring technologies," in Engineering in Medicine and Biology Society (EMBC), 2010 annual international conference of the IEEE. IEEE, 2010, pp. 4456-4459.

[11] M. Patel, S. Lal, D. Kavanagh, and P. Rossiter, "Applying neural network analysis on heart rate variability data to assess driver fatigue," Expert systems with Applications, vol. 38, no. 6, pp. 7235-7242, 2011.

[12] J. Sztajzel et al., "Heart rate variability: a noninvasive electrocardiographic method to measure the autonomic nervous system," Swiss medical weekly, vol. 134, no. 35-36, pp. 514-522, 2004.

[13] D. Dawson and K. Reid, "Fatigue, alcohol and performance impairment," Nature, vol. 388, no. 6639, p. 235, 1997.

[14] P. Philip, F. Vervialle, P. Le Breton, J. Taillard, and J. A. Horne, "Fatigue, alcohol, and serious road crashes in france: factorial study of national data," Bmj, vol. 322, no. 7290, pp. 829-830, 2001.

[15] CardioID. (2017, June) Oficial site of CardioWheel (CardioID), [Online]. Available: https://www.cardio-id.com/cardiowheel

[16] M. G. Hinchey, R. Sterritt, and C. Rouff, "Swarms and swarm intelligence," Computer, vol. 40, no. 4, 2007.

[17] C. W. Reynolds, "Steering behaviors for autonomous characters," in Game developers conference, vol. 1999, 1999, pp. 763-782.

[18] D. Shiffman, S. Fry, and Z. Marsh, The nature of code. D. Shiffman, 2012.

[19] M. Wright, "Open sound control: an enabling technology for musical networking," Organised Sound, vol. 10, no. 3, p. 193-200, 2005.

[20] Microsoft. (2004, June) Integration topologies: Publish/subscribe pattern. [Online]. Available: https://msdn.microsoft.com/enus/library/ff649664.aspx 